\title{
Nonlinear Subspace Clustering Using Curvature Constrained Distances
}

\author{
Amir Babaeian $^{\mathrm{a}, * *}$, Mohammadreaza Babaee $^{\mathrm{b}}$, Alireza Bayestehtashk ${ }^{\mathrm{c}}$, Mojtaba Bandarabadi $^{\mathrm{d}}$ \\ ${ }^{a}$ University of California San Diego, San Diego, California,USA \\ ${ }^{b}$ Technische Universität München, Munich, Geramany \\ ${ }^{c}$ Oregon Health $\mathcal{E}$ Science University, Portland, Oregon, USA \\ ${ }^{d}$ University of Coimbra, Coimbra, Portugal
}

\section{ABSTRACT}

The massive amount of high-dimensional data in science and engineering demands new trends in data analysis. Subspace techniques have shown remarkable success in numerous problems in computer vision and data mining, where the goal is to recover the low-dimensional structure of data in an ambient space. Traditional subspace methods like PCA and ICA assume that the data is coming from a single manifold. However, the data might come from several (possibly intersected) manifolds (surfaces). This has caused the development of new nonlinear techniques to cluster subspaces of high-dimensional data. In this paper, we propose a new algorithm for subspace clustering of data, where the data consists of several possibly intersected manifolds. To this end, we first propose a curvature constraint to find the shortest path between data points and then use it in Isomap for subspace learning. The algorithm chooses several landmark nodes at random and then checks whether there is a curvature constrained path between each landmark node and all other nodes in the neighborhood graph. It builds a binary feature vector for each point where each entry represents the the connectivity of that point to a particular landmark. Then the binary feature vectors could be used as a input of conventional clustering algorithms such as hierarchical clustering. The performed experiments on both synthetic and real data sets confirm the performance of our algorithm.

(c) 2015 Elsevier Ltd. All rights reserved.

\section{Introduction}

The amount of collected data has been increasing exponentially over the last decade. In many areas of machine learning, computer vision and data analysis, the data is represented by very high-dimensional features. For instance, images and videos are represented by millions of pixels. Therefore, the computational complexity of the data is a dramatic challenge in data processing which is referred to the "curse of dimensionality". However, high-dimensional data in most cases comes from low-dimensional structures instead of been uniformly distributed in ambient space (40). Hence, many techniques have been proposed recently to recover the low-dimensional structure of the data from ambient space, the so-called subspace learning. Some work assume that the data is coming from a single structure like manifold learning techniques (40). On the other hand, some methods assume that the data is coming from several structures.

In this paper, we propose a novel technique in recovering a low-dimensional representation of the data coming from several (possibly) intersected structures (surfaces). Therefore, the problem is multi-manifold clustering, which aims to label each data point according to the surface it comes from. This problem may exist in a number of applications, such as the extraction of galaxy clusters (24), road tracking (14), and target tracking $(5 ; 4 ; 28 ; 2 ; 3)$. For instance, in motion segmentation $(22 ; 13 ; 36)$ and face recognition $(20 ; 6 ; 12)$, the underlying surfaces are usually assumed to be linear or affine. In our approach, the main assumption is that the surfaces are smooth. In Fig. 1, the input and output of our algorithm is depicted. Here, we assume that the data is coming from three smooth manifolds (left) and the goal is to distinguish them with different labels/colors (left).

\footnotetext{
${ }^{* *}$ Corresponding author:

e-mail: ababaeian@ucsd.edu (Amir Babaeian)
} 


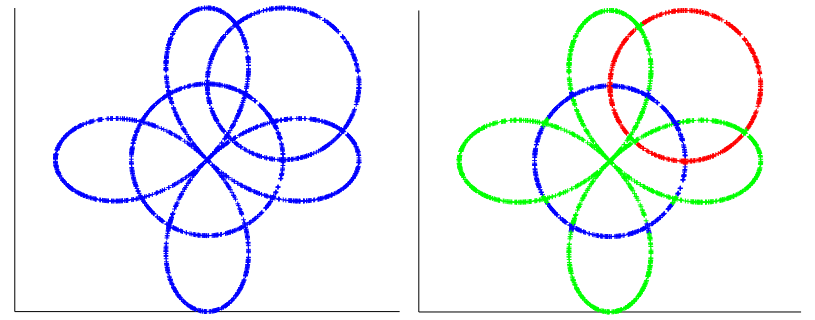

Fig. 1. Simulated data illustrating the problem of multi-manifold clustering. Left: Input data; Right: Output data obtained by our method.

Several techniques have been proposed for multiple manifold clustering. However, most methods are designed for the case where manifolds do not intersect $(25 ; 11)$ or the manifolds that intersect have different either intrinsic dimensions or densities $(16 ; 1)$. Basically, there are a few approaches aiming to recover the intersected manifolds. For instance, Souvenir et al. (31) implement a variant of $\mathrm{K}$-means $(7 ; 8 ; 32)$ where the cluster centers are considered as manifolds. Guo et al. (19) propose to minimize a (combinatorial) energy that includes local orientation information by using a tabu search. Recently, the state-ofthe-art methods are based on local Principal Component Analysis (PCA). For example, the multi-scale spectral method of (21) uses the clustering routine of (17), which is developed in the context of semi-supervised learning and is inspired by the works of (38) and (18).

We propose a remarkably different approach for the problem of clustering multiple intersected manifolds based on connecting points to landmarks via curvature-constrained paths. Our approach can be interpreted as a constrained variant of (33), where we utilize a constrained shortest path distances. Isomap has been specifically designed for dimensionality reduction (or manifold learning) in a single-manifold setting used in different applications $(35 ; 39 ; 26)$. However, in particular, it cannot handle a self intersecting manifold. In our approach, the curvature constraint on paths prevents connecting points from one cluster to points from a different intersecting cluster. The algorithm is implemented as a simple variation of Dijkstra's algorithm.

The rest of the paper is organized as follows. In Section 2, we provide the related work in the area of multiple manifold clustering. Section 3.2 explains the notion of curvature constrained shortest-path and it's connection to the curvature constrained shortest-path. In Section 4, we introduce our algorithm for multi-manifold clustering. In section 5, we provide the detailed information about the performed experiments on both synthetic and real data sets. There we compare the performance of our algorithm with several other algorithm. Additionally, we discuss the robustness of our method to noise. We conclude our paper and provide an outline for future work in Section 6.

\section{Related Works}

The last decade saw a flurry of propositions aiming at highdimensional data clustering when the underlying clusters are not convex and particularly, in the situation where the points are sampled near low-dimensional structures. In the previous section, a few related works were introduced and now in this section, we elaborate on three of them, namely K-Manifolds (KM) (31), Spectral Curvature Clustering (SCC) (10), and Spectral Multi-Manifold Clustering (SMMC) (38). Furthermore, we use them as benchmarks in our experiments. Our choice was dictated by performance, code availability and relevance to our particular setting.

The method of (21) renders impressive results but is hard to tune and relies on many parameters. The method of (18) is very similar to that of (38) and the code was not publicly available at the moment of writing this paper. The other methods for multimanifold clustering (to the best of our knowledge) were not designed to resolve intersections of clusters of possibly identical intrinsic dimensions and sampling densities. Therefore, we chose the subspace clustering method of (10) among a few others methods that perform well in this context.

\subsection{K-Manifolds}

Souvenir et al. (31) suggest an algorithm that mimics K-means by replacing centroid points with centroid submanifolds. The method starts like Isomap by building a neighborhood graph and computing shortest path distances within the graph. After randomly initializing a $K$-by- $n$ weight matrix, $W=\left(w_{k i}\right)$, where $w_{k i}$ represents the probability that point $i$ belongs to the $k$ th cluster, it alternates between an M-Step and an E-Step. In the M-Step, for each $k$, the points are embedded in $\mathbb{R}^{K}$ using a weighted variant of multidimensional scaling using the weights $\left(w_{k i}: i=1, \ldots, n\right)$. In the E-Step, for each $k$ and $i$, the normal distance of point $x_{i}$ to the cluster $k$ is estimated as

$$
\delta_{k i}=\frac{\sum_{j} w_{k j}\left(d\left(x_{i}, x_{j}\right)-d_{k}\left(x_{i}, x_{j}\right)\right)}{\sum_{j} w_{k j}}
$$

where $d\left(x_{i}, x_{j}\right)$ denotes the shortest path distance in the neighborhood graph and $d_{k}\left(x_{i}, x_{j}\right)$ denotes the Euclidean distance in the $k$ th embedding, between points $x_{i}$ and $x_{j}$. The weights are then updated as $w_{k i} \propto \exp \left(-d_{k i}^{2} / \sigma^{2}\right)$ such that $\sum_{k} w_{k i}=1$ for all $i$, where $\sigma^{2}$ is chosen automatically.

\subsection{Spectral Curvature Clustering}

Chen et al. (10) propose a spectral method for subspace clustering based on the assumption that the underlying surfaces are affine. However, we compare our method to theirs when the surfaces are affine and also when the surfaces are curved. The latter is done as a proof of concept, whereas it is clear that this method cannot handle curved surfaces, like any other method for subspace clustering. The procedure assumes that all subspaces are of the same dimension $d$, which is a parameter of the method. For each $(d+2)$-tuple, $x_{i_{1}}, \ldots, x_{i_{d+2}}$, it computes a notion of curvature $C_{i_{1}, \ldots, i_{d+2}}$ which measure how well this $(d+2)$ tuple is approximated by an affine subspace of dimension $d$. After reducing the tensor $\mathbf{C}=\left(C_{i_{1}, \ldots, i_{d+2}}: i_{t}=1, \ldots, N\right)$ spectral graph partitioning (25) is applied. 


\subsection{Spectral Multi-Manifold Clustering}

Wang et al. (38) propose a spectral clustering method using a dissimilarity function that factors in the Euclidean distance and the discrepancy between the local orientation of the data points. The surfaces are assumed to be of the same dimension $d$ and this number should be known as a prior. First, a mixture of probabilistic principal component analyzers (34) are fitted to the data, approximating the point cloud by a union of $d$-planes. This is used to estimate the tangent subspace at each data point. The dissimilarity between two data points is then an increasing function of their Euclidean distance and also the principal angles between their respective affine subspaces. These dissimilarities are fed into the spectral graph partitioning method proposed by (25).

\subsection{Spectral Clustering}

Spectral clustering has been widely used as a clustering method for high-dimensional data. First, it forms a similarity matrix of the points where elements of this matrix are the relative similarity between each pair of points in a highdimensional space. Then it computes the normalized or unnormalized Laplacian matrix $L$. By computing the first $K$ eigenvectors of $L$ we form a new matrix $U$, where the number of columns of $U$ is equal to $K$. The rows of $U$ are the low-dimensional representation of high-dimensional data. By applying the k-means algorithm to the rows of $U$ we extract the cluster membership for each point. Spectral clustering is closely related to the nonlinear dimensionality reduction methods like locally-linear embedding.

\section{Constrained path}

\subsection{Curvature constraint}

Considering that all the subspaces are flat, we can use the fact that the smooth nonlinear subspaces are locally isomorphic to the euclidean space. Given data points $x_{1}, \ldots, x_{N}$, We consider two types of neighborhood graph (23):

- $\varepsilon$-ball. $x_{i}$ and $x_{j}$ are connected if $\left\|x_{i}-x_{j}\right\| \leq \varepsilon$, where $\|\cdot\|$ denotes the Euclidean norm.

- k-nearest neighbor. $x_{i}$ and $x_{j}$ are connected if $x_{j}$ is among the $k$-nearest neighbors of $x_{i}$ (in the Euclidean metric), or vice-versa.

The main contribution of this paper is to use a constraint to avoid connecting points that belong to different subspaces. The paths are constrained in order to control their smoothness. In other words, constrained shortest path is used to approximate the geodesic distance between points. The ratio of a constrained to an unconstrained shortest path between two points should be close to one if points belong to the same surface. For an ordered triplet of points $(x, y, z)$ in $\mathbb{R}^{D}$, we define the curvature as

$$
\operatorname{curv}(x, y, z)= \begin{cases}(R(x, y, z))^{-1}, & \text { if } \angle(x, y, z)<\frac{\pi}{2}, \\ \infty, & \text { otherwise }\end{cases}
$$

where $\angle$ stands for the angle and $R(x, y, z)$ is the radius of the circle passing through $x, y, z$.

$$
R(x, y, z)=\frac{\sqrt{\begin{array}{c}
\|x-y\|^{2}+\|z-y\|^{2} \\
+2\|x-y\|\|z-y\| \cos \angle(x, y, z)
\end{array}}}{\sin \angle(x, y, z)} .
$$

with $R(x, y, z)=\infty$ if $x, y, z$ are aligned.

Definition. For a curvature $\kappa>0$, we say that a path $\left(x_{i_{1}}, \ldots, x_{i_{m}}\right)$ is $\kappa$-constrained if $\operatorname{curv}\left(x_{i_{t-1}}, x_{i_{t}}, x_{i_{t+1}}\right) \leq \kappa, \quad \forall t=$ $2, \ldots, m-1$.

\subsection{Angle constraint}

For an ordered triplet of points $(x, y, z)$ in $\mathbb{R}^{D}$, define its angle as,

$$
\begin{array}{r}
\angle(x, y, z)=\angle(\overrightarrow{x y}, \overrightarrow{y z})=\cos ^{-1}\left(\frac{\langle y-x, z-y\rangle}{\|y-x\|\|z-y\|}\right) \\
\in[0, \pi]
\end{array}
$$

Definition. We say that a sequence of points $\left(x_{i_{1}}, \ldots, x_{i_{m}}\right)$ is $\theta$-angle constrained if the angles between successive segments are all bounded by $\theta$, meaning

$$
\angle\left(x_{i_{t-1}}, x_{i_{t}}, x_{i_{t+1}}\right) \leq \theta, \quad \forall t=2, \ldots, m-1 .
$$

We use a modified version of a shortest path algorithm to compute the constrained path between a source point and other nodes in the neighborhood graph (see algorithm 1 below). The main difference between our algorithm and a shortest path is to check the constraint between three successive points during the process of Dijkstra's algorithm. This simple modification of Dijkstra's algorithm allows us to compute the constrained shortest path between points with the same computational cost as Dijkstra.

\section{Subspace Clustering}

Having introduced our constrained shortest path distances, we address the problem of multiple surface (manifold) clustering. Given a sample $x_{1}, \ldots, x_{n} \in \mathbb{R}^{D}$ sampled from $\mathcal{S}_{1} \cup \cdots \cup \mathcal{S}_{K}$, where for each $k, \mathcal{S}_{k}$ is a smooth, but possibly self-intersecting surface, we aim at labeling each point according to the surface it belongs to. Our algorithm is quite distinct from all the other methods for multi-manifold clustering we are aware of, although it also starts by building a $q$-nearest neighbor graph like many others. The main idea is to cluster points that are connected by an angle-constrained path in the neighborhood graph. We assume two surfaces $S_{1}$ and $S_{2}$ intersect at a strictly positive angle. Then for the 'most' pairs of data points $x_{i_{1}} \in S_{1}$ and $x_{i_{2}} \in S_{2}$, a path in the graph going from $x_{i_{1}}$ to $x_{i_{2}}$ has at least one large angle between two successive edges in order of the incidence angle between the surfaces. Additionally, for the 'most' pairs of data points $x_{i_{1}}, x_{i_{2}} \in S_{1}$, there is a path with all angles between successive relatively small edges. To speedup the implementation, we select $M$ landmarks (with $M$ slightly larger than $K$ ) at random among the data points and only determine which data points are connected to which landmark via a $\kappa$-constrained path in the graph. This means $M$ and $\kappa$ are the parameters of the algorithm. Let $\xi_{\ell i}=1$ denotes that the point $i$ 


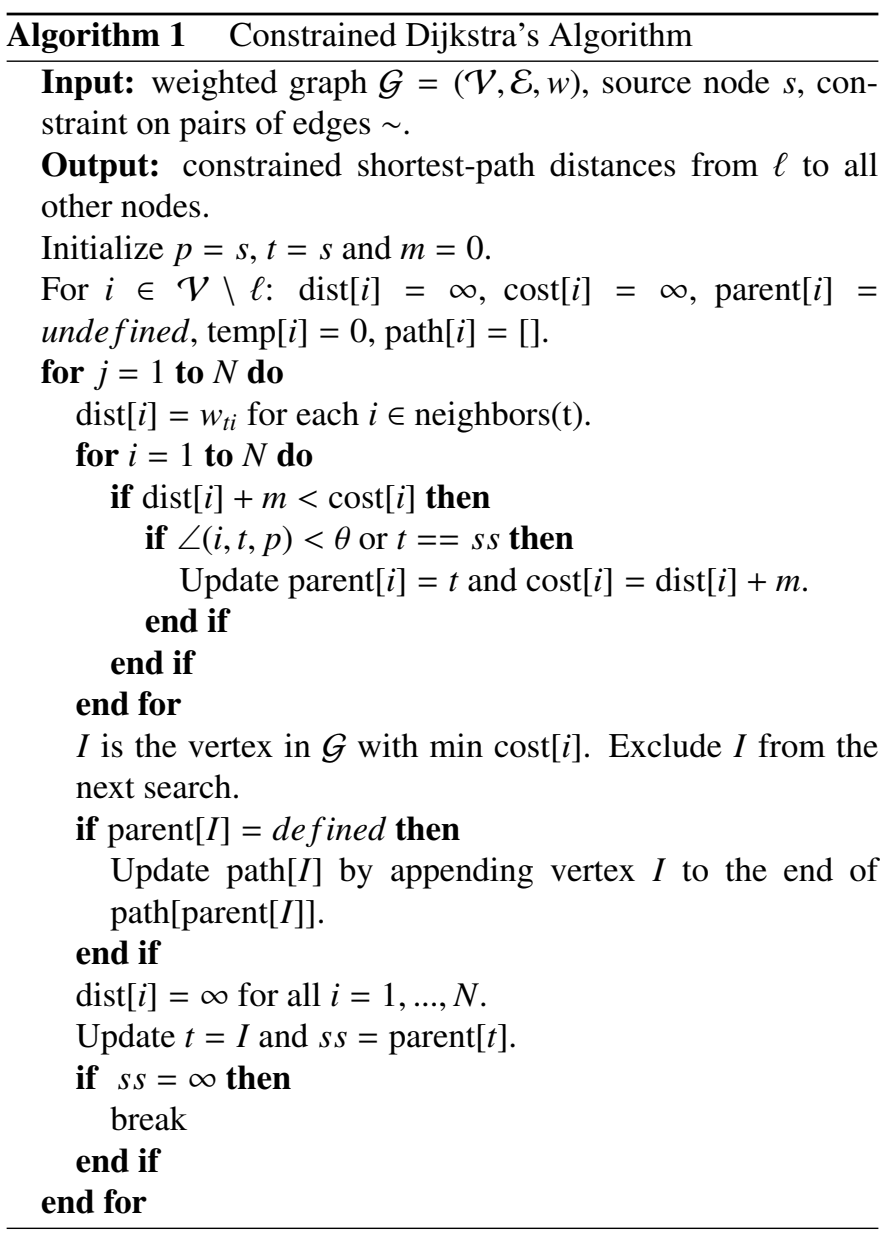

and the landmark $\ell$ are connected and $\xi_{\ell i}=0$ if not. Then, we use $\xi_{i}:=\left(\xi_{\ell i}: \ell=1, \ldots, M\right)$ as feature vectors that we cluster using hierarchical clustering with complete linkage in order to determine the label of data points.

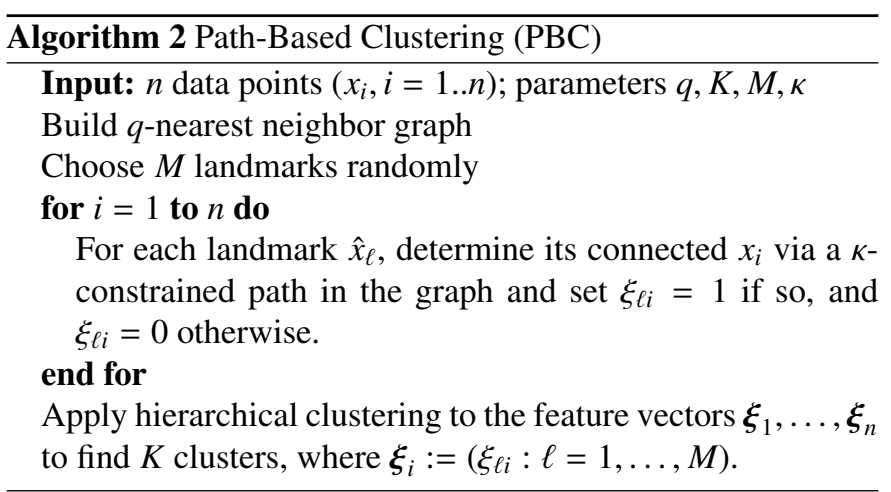

\subsection{Intersections}

We are mainly interested in the case where the surfaces intersect. Concretely, given $K$ compact and simply connected submanifolds $S_{1}, \ldots, S_{K} \subset \mathbb{R}^{D}$ of maximum pointwise curvature bounded by $\kappa<\infty$, we consider the noisy mixture distribution

$$
x=s+z, \quad s \sim \sum_{k=1}^{K} \pi_{k} \mu_{S_{k}} \quad z \sim \mu_{B(0, \tau)},
$$

where $\mu_{S}$ denotes the uniform distribution over the set $S$.

Definition. We consider two smooth sub-manifolds $S_{1}$ and $S_{2}$ having an incidence angle $\alpha \in(0, \pi / 2)$ at an intersection point $x \in S_{1} \cap S_{2}$ if the smallest nonzero principal angle between the tangent subspaces of $S_{1}$ and $S_{2}$ at $x$ is equal to $\alpha$.

We assume that for any pair of underlying surfaces $S_{k}$ and $S_{\ell}$, the minimum incidence angle between them at any point along their intersection is at least $\alpha>0$. The compactness of the surfaces imply the existence of a function $\omega(\varepsilon) \rightarrow 0$ with $\varepsilon \rightarrow 0$ such that for any $\varepsilon>0$, if $\operatorname{dist}\left(x, S_{k}\right) \vee \operatorname{dist}\left(x, S_{\ell}\right) \leq \varepsilon$, then $\operatorname{dist}\left(x, S_{k} \cap S_{\ell}\right) \leq \omega(\varepsilon)$. Otherwise, there is $C>0$ such that for all $m \geq 1$, there is $x_{t}$ satisfying $\operatorname{dist}\left(x_{t}, S_{k}\right) \vee \operatorname{dist}\left(x_{t}, S_{\ell}\right) \leq 1 / m$ and $\operatorname{dist}\left(x_{t}, S_{k} \cap S_{\ell}\right) \geq C$. By the fact that $\left(x_{t}\right)$ is bounded (since $S_{k}$ and $S_{\ell}$ are), there is a subsequence that converges to some $x$, which is necessarily in both $S_{k}$ and $S_{\ell}$ since these sets are closed. At the same time, $\operatorname{dist}\left(x, S_{k} \cap S_{\ell}\right) \geq C$ by continuity of the distance function, which is a contradiction. In fact, the assumption on the incidence angle implies that $\omega(\varepsilon) \leq C \varepsilon$ for some $C>0$ not depending on $\varepsilon$, but this is much longer to prove.

\subsection{Landmarks}

A key ingredient to the success of the procedure is that there is at least one landmark chosen from each cluster that is far away from any other clusters. This means we work with a stronger condition that all chosen landmarks are more than $\varepsilon$ away from other clusters, which actually leads to simplifications later on in the discussion. The probability of selecting a point from (5) with $s \in S_{k}$ away from other clusters by at least $\varepsilon$ is $p_{k}(\varepsilon):=\pi_{k}\left(1-\mu_{S_{k}}\left(\cup_{\ell \neq k} S_{\ell}^{\varepsilon}\right)\right)$, where $S^{\varepsilon}$ denotes the points in $\mathbb{R}^{D}$ within the distance $\varepsilon$ of $S$. Therefore, the probability that all $M$ landmarks are at least $\varepsilon$ away from other clusters is equal to

$$
p_{M}^{*}(\varepsilon+2 \tau):=\left(\sum_{k} p_{k}(\varepsilon+2 \tau)\right)^{M},
$$

where we used the triangle inequality. When $\varepsilon, \tau \rightarrow 0, p_{k}(\varepsilon+$ $2 \tau) \rightarrow \pi_{k}\left(1-\mu_{S_{k}}\left(\cup_{\ell \neq k} S_{\ell}\right)\right)=\pi_{k}$, implying $p_{M}^{*}(\varepsilon+2 \tau) \rightarrow 1$, if we assume that $\mu_{S_{k}}\left(S_{\ell}\right)=0$ when $\ell \neq k$, which is a very mild assumption we make hereafter. This result shows that one landmark per class would be enough to guarantee the capture of correct subspaces.

\subsection{Robustness to Noise}

We note that all the other known methods for multi-manifold clustering do not perform well unless the noise level is quite small. As it appears in our method, by increasing the amount of noise, the possibility of connecting points from different surfaces with a curvature constrained path increases. Fig. 2 shows the error rate of two intersecting curves over the amount of standard uniform noise. As it can be illustrated, by increasing the amount of noise, the notion of two different surfaces (manifolds) would be ambiguous such that we can say all the points belong to one manifold. All other methods fail to capture the correct manifold with even small amounts of noise where our method still performs well with $20 \%$ noise. 


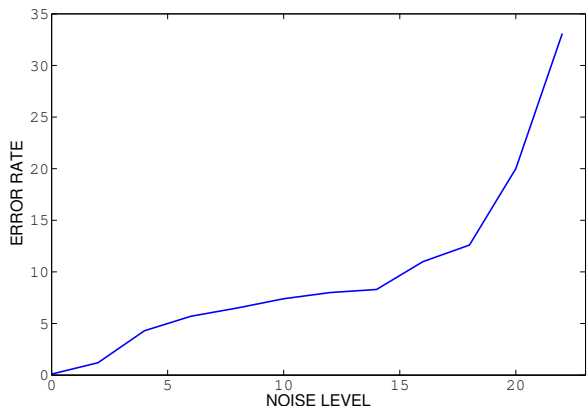

Fig. 2. Effect of noise on the performance of our algorithm on the Two Curves data set (the data set is introduced in experimental section).

\subsection{The choice of constraint}

Perhaps the main challenge of our algorithm is the choice of the angle constraint within the similarity graph. In one hand, a large angle value for this constraint causes the points from different manifolds to be connected using a constrained shortestpath. This ultimately leads to multiple manifolds being clustered as one class. On the other hand, choosing a small value does not allow us to accurately capture the structure of the manifold. However, we start with an angle constraint of $50^{\circ}$ and use $1 \%$ of the points in each cluster as labeled data. We then measure the performance of our algorithm on the labeled data. In order to find the optimum angle constraint, we increase or decreased our angle constraint by a certain factor. We initially begin with dividing our angle constraint by a factor of 2 , until the error starts decreasing. In the case that the error increases, we increase the angle constraint by a factor of $4 / 3$. In most cases, we were able to find the optimal angle constraint within 5 iterations.

\subsection{Computational Complexity}

Building a symmetric $q$-nearest neighbor graph using cover trees (9) takes order $O(q N \log N)$, where the implicit constant depends exponentially on the intrinsic dimensions of the surfaces and linearly on the ambient dimension $D$. The angleconstrained pathfinder routine is a simple variant of Dijkstra's algorithm, whose implementation by Fibonacci heaps runs in $O(q N \log N)$. Hence, calling this routine once for each landmark costs $O(q M N \log N)$. Grouping the feature vectors takes order $O(F M N)$ and then clustering them by complete linkage costs $O\left(F^{2} \log F\right)$, where $F$ is the (data-dependent) number of distinct feature vectors $\xi_{i}$, which is often of the order of $K$ in our experiments.

\subsection{Relation to Landmark-Isomap}

As (27) argues that Local Linear Embedding (LLE) (29) could be used for clustering non-intersecting manifolds, in a sense, we showed that a constrained version of the LandmarkIsomap (30) can be used to cluster possibly intersecting manifolds

\section{Numerical Experiments}

In order to assess the quality of our proposed method, we applied our method to both synthetic and real data sets and compared the results with the output of others. In this section, we explain the experimental results.

\subsection{Synthetic Data}

The synthetic data sets we generated are similar to those appearing in the literature. We have applied our method to 6 different synthetic data sets including: 1) Cone and plane $(C P)$; 2) Cross (CR); 3) Rose and rose (RR); 4) Two arcs (TA); 5) Two curves (TC) and 6) Two spheres (TS) and their results are depicted in Fig. 3-Fig. 8, respectively. We compared the performance of our algorithm (PBC) with four other techniques, namely Spectral Multi-Manifolds Clustering (SMMC), K-Manifolds (KM), Spectral Curvature Clustering (SCC), and Spectra,1 Clustering (SC). The details of these algorithms are provided in Section 2. As the results show, SCC works well on linear manifolds (i.e. Cross and Two curves) as expected, while it fails when the manifolds are not linear and have a curvature like in Fig. 3e. KM fails in the more complicated examples like in Fig. 5d and Fig. 8d. Additionally, we found out that the $\mathrm{KM}$ algorithm is extremely slow in comparison to other used algorithms since it has to compute the shortest path between all points. Therefore, this algorithm is not applicable in dealing with large data sets. Moreover, it assumes that the manifolds have intersection, and otherwise it does not work properly. Our method and SMMC perform comparably on most datasets, but SMMC fails in the Two curves data set (See Fig. 7c). Finally, the SC algorithm delivers the worst results in all data sets. In the end, KM, SCC, and SMMC all relied on the fact that all surfaces have the same dimension and it should be known in advance, which is a big assumption. In contrary, our method does not need any knowledge of the intrinsic dimensions of the surfaces and can operate even when they are different.

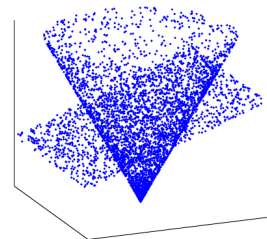

(a) Original data

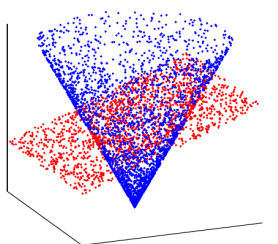

(d) $\mathrm{KM}$

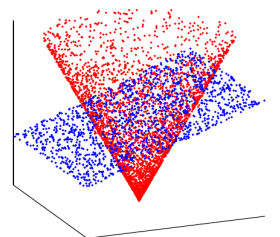

(b) $\mathrm{PBC}$

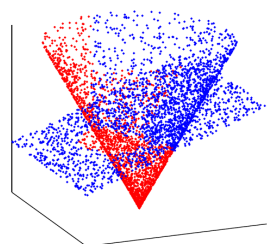

(e) SCC

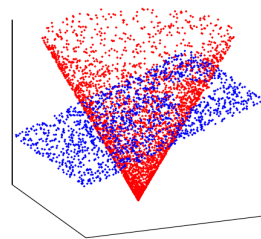

(c) SMMC

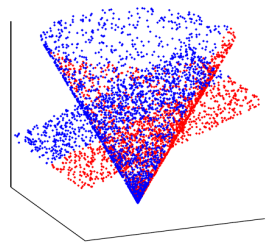

(f) $\mathrm{SC}$
Fig. 3. clustering results for Cone and plane data set

The parameters of our techniques for these six data sets are presented in Table. 5.1 


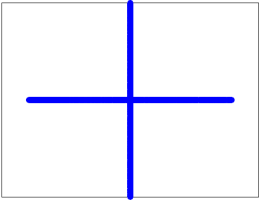

(a) Original data

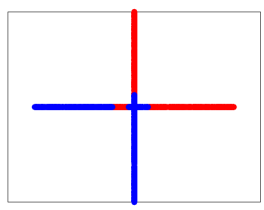

(d) $\mathrm{KM}$

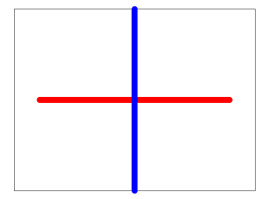

(b) PBC

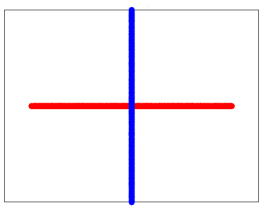

(e) SCC

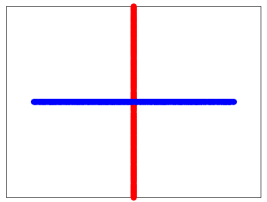

(c) SMMC

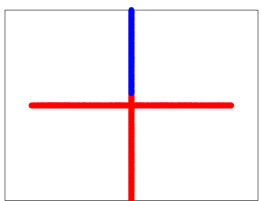

(f) $\mathrm{SC}$

Fig. 4. clustering results for Cross data set

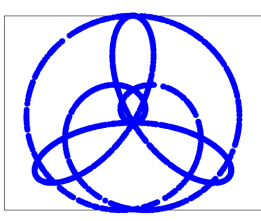

(a) Original data

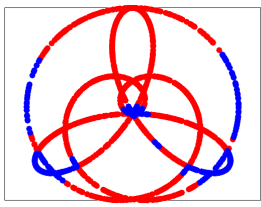

(d) $\mathrm{KM}$

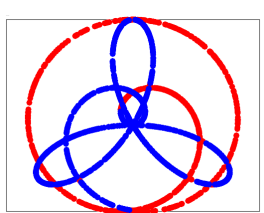

(b) PBC

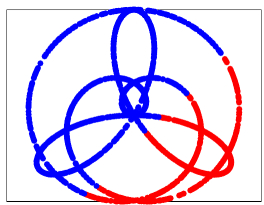

(e) SCC

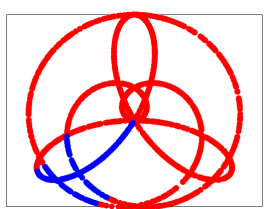

(c) SMMC

Fig. 5. clustering results for Rose and rose data set

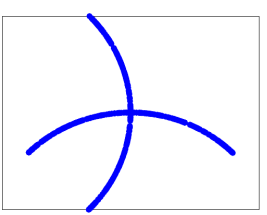

(a) Original data

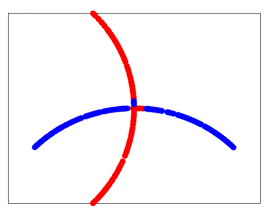

(d) $\mathrm{KM}$

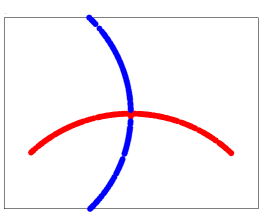

(b) PBC

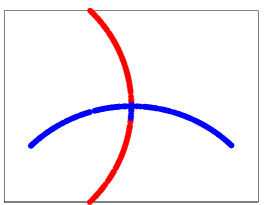

(e) $\mathrm{SCC}$

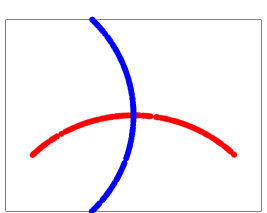

(c) SMMC

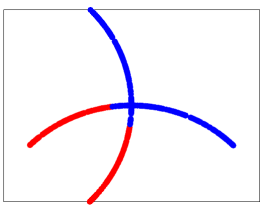

(f) $\mathrm{SC}$

Fig. 6. clustering results for Two arcs data set

\subsection{Real Data}

\subsubsection{Clustering of 2D Image Data Set}

We use a subset of the Caltech101 data $\operatorname{set}^{1}$ as our real data consisting 3500 images categorized in 10 different classes (see Fig. 10). We utilized a Bag-of-words model of SIFT features to represent the content of each image. The feature vectors have the size of 128. Then, we applied PCA on the feature vectors

\footnotetext{
${ }^{1}$ http://www.vision.caltech.edu/Image_Datasets/Caltech101
}

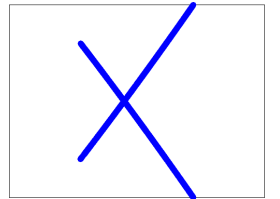

(a) Original data

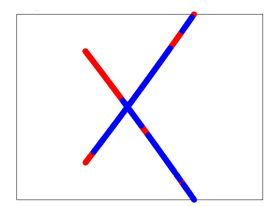

(d) KM

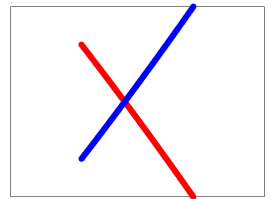

(b) PBC

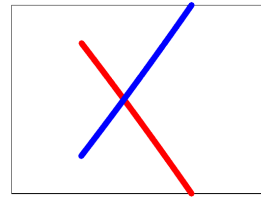

(e) SCC (c) SMMC

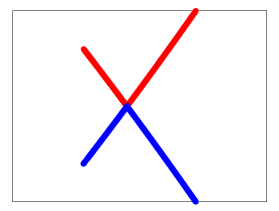

(f) $\mathrm{SC}$

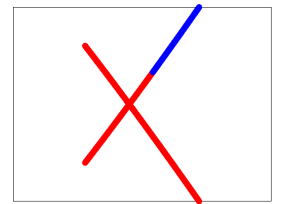

Fig. 7. clustering results for Two curves data set

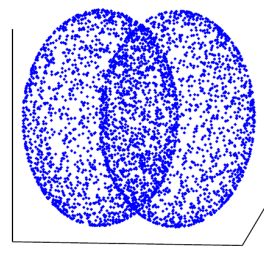

(a) Original data

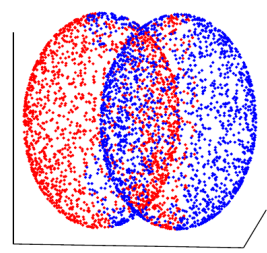

(d) KM

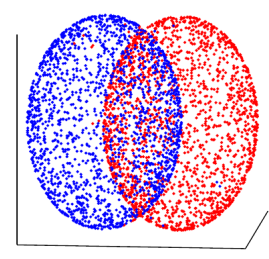

(b) PBC

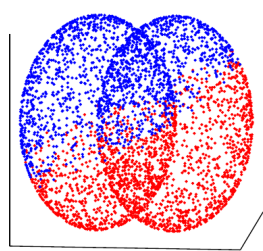

(e) SCC

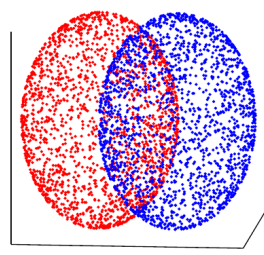

(c) SMMC

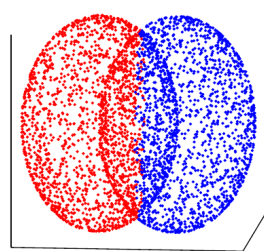

(f) $\mathrm{SC}$
Fig. 8. clustering results for Two spheres data set
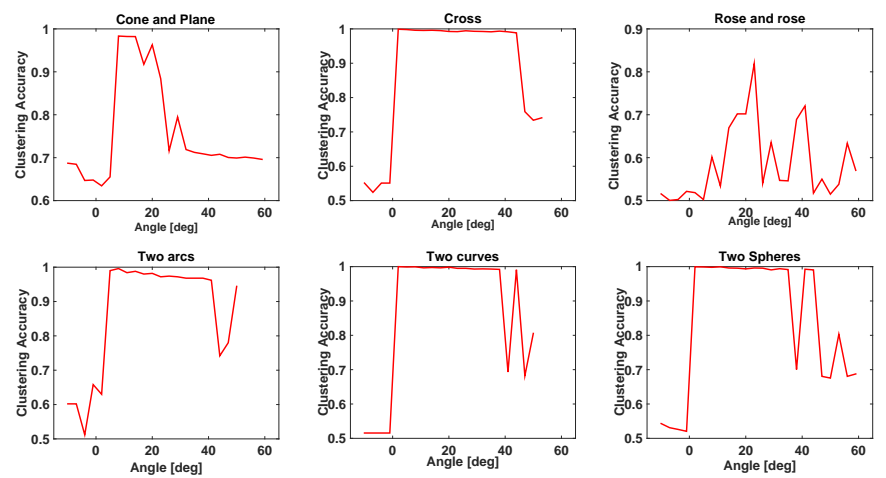

Fig. 9. The sensivity of six synthetic data sets to the angle constraint.

to get smaller features with the size of 10 . This was mainly done for speeding up the computations. Here again we applied the used methods and measured the clustering accuracy. However, we did this experiment with a different number of classes, namely $2,4,6,8$, and 10 . Precisely, each time we selected K classes from the data set, applied the methods and measured the accuracy. The clustering results for different $\mathrm{K}$ are presented in Table. 2 . 


\begin{tabular}{|c||c|c|c|c|c|c|}
\hline Parameter & CP & CR & RR & TA & TC & TS \\
\hline \hline epsilon & 80 & 50 & 30 & 60 & 30 & 40 \\
angle & 15 & 10 & 10 & 5 & 20 & 15 \\
clusters & 2 & 2 & 2 & 2 & 2 & 2 \\
landmarks & 100 & 3 & 20 & 10 & 2 & 60 \\
\hline \hline accuracy & 0.983 & 0.996 & 0.8015 & 0.986 & 0.997 & 0.965 \\
\hline
\end{tabular}

Table 1. Parameters of PBC algorithm applied to six synthetic data sets.

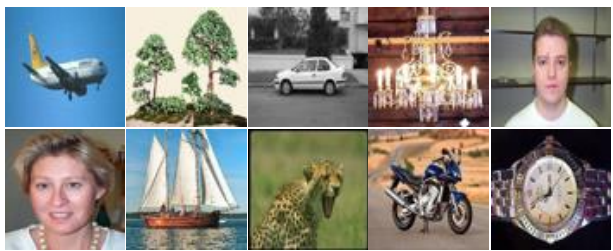

Fig. 10. Some sample images of Caltech data set

\subsection{Clustering of Human Motion Sequences}

In computer vision clustering of human motion sequences into different classes of activities performed by a subject is referred to a temporal segmentation. In this section we test our algorithm on a sequence of video frames including different activities performed by a subject. We choose 4 mixed actions from subject 86, trial number 9 of the CMU MoCap dataset. The data consists of a temporal sequence with a 62-dimensional representation of the human body via markers in $\mathbb{R}^{3}$. One motion sequence of 4794 frames and the corresponding result of path-based multi-manifold clustering are given in Fig. 11. Four activities are labeled from 1 to 4 . We do not label the frames where the subject switches from one action to another because of the uncertainty about the true activity.
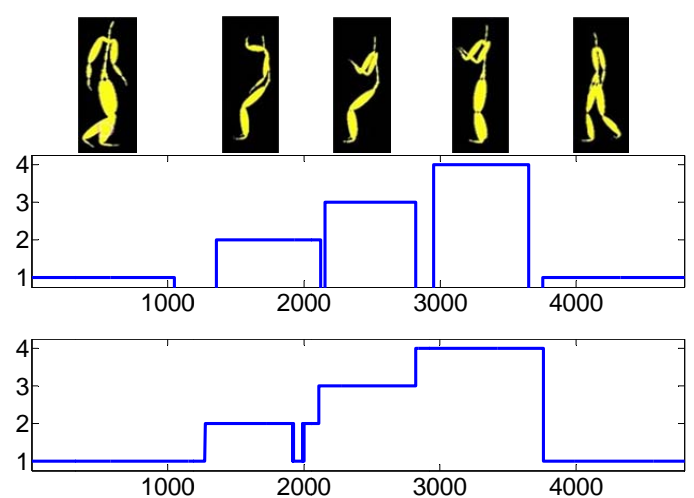

Fig. 11. Result of human activity segmentation using Path-Based Clustering. There are 4 activities: walking (1), looking (2), sitting (3) and standing (4). Top: a sample of the sequence. Middle: ground truth. Bottom: output of our algorithm.

\subsection{Face Clustering}

In this section we consider the problem of clustering faces under varying illumination conditions. We consider the same data set as used in $(10 ; 37)$. This is the subset of the Yale Face Database B (15) that contains the frontal face image of three different subjects $(5,8,10)$ (see Fig. 12). This images has been taken under 64 different illumination conditions. In total we have $64 \times 3$ face images and we down sample each image to

\begin{tabular}{|c||c|c|c|c|c|}
\hline K & SCC & SC & KM & SMMC & PBC \\
\hline \hline 2 & 0.6846 & 0.7456 & - & $\mathbf{0 . 7 7 0 6}$ & 0.7387 \\
& \pm 0.1417 & \pm 0.1569 & & $\pm \mathbf{0 . 1 5 1 8}$ & \pm 0.1654 \\
\hline 4 & 0.5694 & 0.5621 & - & 0.5598 & $\mathbf{0 . 5 7 8 2}$ \\
& \pm 0.0890 & \pm 0.1063 & & \pm 0.0996 & $\pm \mathbf{0 . 1 1 5 7}$ \\
\hline 6 & 0.4898 & 0.4927 & - & 0.4139 & $\mathbf{0 . 5 1 8 0}$ \\
& \pm 0.0514 & \pm 0.0643 & & \pm 0.0936 & $\pm \mathbf{0 . 0 6 8 9}$ \\
\hline 8 & 0.4074 & 0.4122 & - & 0.4252 & $\mathbf{0 . 4 4 5 9}$ \\
& \pm 0.0751 & \pm 0.0111 & & \pm 0.0489 & $\pm \mathbf{0 . 0 5 6 9}$ \\
\hline 10 & 0.4074 & 0.4122 & - & 0.4252 & $\mathbf{0 . 4 4 5 9}$ \\
& \pm 0.0751 & \pm 0.0111 & & \pm 0.0489 & $\pm \mathbf{0 . 0 5 6 9}$ \\
\hline
\end{tabular}

Table 2. Clustering accuracy of multiple subjects for the caltech data set

$120 \times 160$. After concatenating all pixels of each image and putting them into a vector of size 1920 , we construct a matrix of the size $192 \times 19200$ where each row represents an image of the original sequence related to three subjects. Applying our algorithm on this matrix we get a perfect clustering (100\%). We repeated the experiment, this time projecting the data onto the top 10 principal components as done in $(10 ; 37)$, obtaining a matrix of size $192 \times 10$. We still get a $100 \%$ accuracy, for an even wider range of parameters. This result is comparable with what $(10 ; 37)$ reported in their paper using subspace clustering.

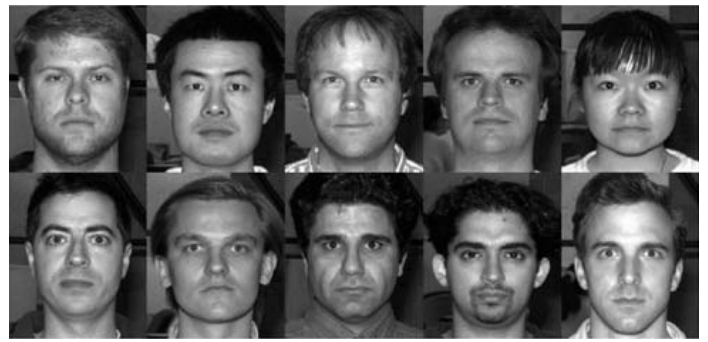

Fig. 12. The ten subjects in the Yale Face Database B. First row: subjects 1 through 5; second row: subjects 6 to 10

\section{Conclusion}

In this paper we proposed a method for clustering multiple subspaces. These subspaces could intersect with each other and come from different manifolds in ambient spaces. Our method uses a constrained version of a shortest path algorithm to decide whether two points are connected to each other or not. we applied our method to synthetic and real data sets and demonstrated that it performs comparably to the best known methods such as K-manifold and spectral multi-manifold clustering. We are currently experimenting with variants - some based on other constraints - that would lead to path-based clustering algorithms that perform in practice at least as well as algorithm 2, and are consistent in the large-sample limit.

\section{Acknowledgments}

This work was supported by a grant from the National Science Foundation (DMS- 09-15160). 


\section{References}

[1] E. Arias-Castro, G. Chen, and G. Lerman. Spectral clustering based on local linear approximations. Electron. J. Statist., 5:1537-1587, 2011.

[2] A. Babaeean, A.B. Tashk, M. Bandarabadi, and S. Rastegar. Target tracking using wavelet features and rvm classifier. In Natural Computation, 2008. ICNC '08. Fourth International Conference on, volume 4, pages 569-572, Oct 2008.

[3] A. Babaeean, A.B. Tashk, F. Barzin, and S.M. Hosseini. Target tracking using mean shift and dynamic directional gradient vector flow. In System Theory, 2008. SSST 2008. 40th Southeastern Symposium on, pages 366370, March 2008.

[4] A. Babaeian, S. Rastegar, M. Bandarabadi, and M. Erza. Modify kernel tracking using an efficient color model and active contour. In System Theory, 2009. SSST 2009. 41st Southeastern Symposium on, pages 5963, March 2009.

[5] A. Babaeian, S. Rastegar, M. Bandarabadi, and M. Rezaei. Mean shiftbased object tracking with multiple features. In System Theory, 2009. SSST 2009. 41 st Southeastern Symposium on, pages 68-72, March 2009.

[6] R. Basri and D. Jacobs. Lambertian reflectance and linear subspaces. IEEE PAMI, 25(2):218-233, 2003.

[7] A. Bayestehtashk and I. Shafran. Parsimonious multivariate copula model for density estimation. In Acoustics, Speech and Signal Processing (ICASSP), 2013 IEEE International Conference on, pages 5750-5754, May 2013.

[8] Alireza Bayestehtashk, Meysam Asgari, Izhak Shafran, and James McNames. Fully automated assessment of the severity of parkinson's disease from speech. Computer Speech $\mathcal{E}$ Language, 2013

[9] Alina Beygelzimer, Sham Kakade, and John Langford. Cover trees for nearest neighbor. In ICML '06: Proceedings of the 23rd international conference on Machine learning, pages 97-104, New York, NY, USA, 2006. ACM

[10] G. Chen and G. Lerman. Spectral curvature clustering (SCC). IJCV, 81(3):317-330, 2009.

[11] Ehsan Elhamifar and Rene Vidal. Sparse manifold clustering and embedding. In J. Shawe-Taylor, R.S. Zemel, P. Bartlett, F.C.N. Pereira, and K.Q. Weinberger, editors, Advances in Neural Information Processing Systems 24, pages 55-63. 2011.

[12] R. Epstein, P. Hallinan, and A. Yuille. $5 \pm 2$ eigenimages suffice: An empirical investigation of low-dimensional lighting models. In IEEE Workshop on Physics-based Modeling in Computer Vision, pages 108-116, June 1995.

[13] Z. Fu, W. Hu, and T. Tan. Similarity based vehicle trajectory clustering and anomaly detection. In ICIP, pages II-602-5, 2005.

[14] D. Geman and B. Jedynak. An active testing model for tracking roads in satellite images. IEEE Trans. Pattern Anal. Mach. Intell., 18:1-14, 1996.

[15] Athinodoros S. Georghiades, Peter N. Belhumeur, and David J. Kriegman. From few to many: Illumination cone models for face recognition under variable lighting and pose. IEEE Trans. Pattern Anal. Mach. Intell., 23(6):643-660, June 2001.

[16] Aristides Gionis, Alexander Hinneburg, Spiros Papadimitriou, and Panayiotis Tsaparas. Dimension induced clustering. In $K D D$ '05: Proceedings of the eleventh ACM SIGKDD international conference on Knowledge discovery in data mining, pages 51-60, New York, NY, USA, 2005. ACM

[17] A.B. Goldberg, X. Zhu, A. Singh, Z. Xu, and R. Nowak. Multi-manifold semi-supervised learning. In AISTATS, pages 169-176, 2009.

[18] Dian Gong, Xuemei Zhao, and Gérard Medioni. Robust multiple manifolds structure learning. In Proc. 29th Intl. Conf. on Machine Learning (ICML), 2012.

[19] Qiyong Guo, Hongyu Li, Wenbin Chen, I-Fan Shen, and Jussi Parkkinen. Manifold clustering via energy minimization. In ICMLA '07: Proceedings of the Sixth International Conference on Machine Learning and Applications, pages 375-380, Washington, DC, USA, 2007. IEEE Computer Society.

[20] J. Ho, M. Yang, J. Lim, K. Lee, and D. Kriegman. Clustering appearances of objects under varying illumination conditions. In $C V P R$, pages 11-18, 2003.

[21] D. Kushnir, M. Galun, and A. Brandt. Fast multiscale clustering and manifold identification. Pattern Recogn., 39(10):1876-1891, 2006

[22] Y. Ma, A. Y. Yang, H. Derksen, and R. Fossum. Estimation of subspace arrangements with applications in modeling and segmenting mixed data. SIAM Review, 50(3):413-458, 2008.
[23] Markus Maier, Matthias Hein, and Ulrike von Luxburg. Optimal construction of k-nearest-neighbor graphs for identifying noisy clusters. Theoretical Computer Science, 410(19):1749-1764, 2009.

[24] V.J. Martínez and E. Saar. Statistics of the Galaxy Distribution. CRC press, Boca Raton, 2002.

[25] A.Y. Ng, M.I. Jordan, and Y. Weiss. On spectral clustering: Analysis and an algorithm. Advances in neural information processing systems, 2:849-856, 2002.

[26] C. Orsenigo and C. Vercellis. An effective double-bounded treeconnected isomap algorithm for microarray data classification. Pattern Recognition Letters, 33(1):9 - 16, 2012.

[27] M. Polito and P. Perona. Grouping and dimensionality reduction by locally linear embedding. Advances in Neural Information Processing Systems, 14:1255-1262, 2001.

[28] S. Rastegar, A. Babaeian, M. Bandarabadi, and Y. Toopchi. Airplane detection and tracking using wavelet features and svm classifier. In System Theory, 2009. SSST 2009. 41 st Southeastern Symposium on, pages 64-67, March 2009.

[29] S. Roweis and L. Saul. Nonlinear dimensionality reduction by locally linear embedding. Science, 290(5500):2323-2326, 2000.

[30] V. Silva and J.B. Tenenbaum. Global versus local methods in nonlinear dimensionality reduction. Advances in neural information processing systems, 15:705-712, 2002.

[31] R. Souvenir and R. Pless. Manifold clustering. In Computer Vision, 2005. ICCV 2005. Tenth IEEE International Conference on, volume 1, pages 648-653 Vol. 1, 2005.

[32] A.R.B. Tashk, A. Sayadiyan, P. Mahale, and M. Nazari. Pattern classiffication using svm with gmm data selection training methode. In Signal Processing and Communications, 2007. ICSPC 2007. IEEE International Conference on, pages 1023-1026, Nov 2007.

[33] J. B. Tenenbaum, V. de Silva, and J. C. Langford. A global geometric framework for nonlinear dimensionality reduction. Science, 290(5500):2319-2323, 2000.

[34] M. Tipping and C. Bishop. Mixtures of probabilistic principal component analysers. Neural Computation, 11(2):443-482, 1999.

[35] Ioannis Tziakos, Andrea Cavallaro, and Li-Qun Xu. Video event segmentation and visualisation in non-linear subspace. Pattern Recognition Letters, 30(2):123 - 131, 2009. Video-based Object and Event Analysis.

[36] R. Vidal and Y. Ma. A unified algebraic approach to 2-D and 3-D motion segmentation and estimation. JMIV, 25(3):403-421, 2006.

[37] R. Vidal, Y. Ma, and S. Sastry. Generalized principal component analysis (gpca). Pattern Analysis and Machine Intelligence, IEEE Transactions on, 27(12):1945-1959, 2005

[38] Y. Wang, Y. Jiang, Y. Wu, and Z.H. Zhou. Spectral clustering on multiple manifolds. Neural Networks, IEEE Transactions on, 22(7):1149-1161, 2011.

[39] Shifeng Weng, Changshui Zhang, and Zhonglin Lin. Exploring the structure of supervised data by discriminant isometric mapping. Pattern Recognition, 38(4):599 - 601, 2005.

[40] C. Yang, D. Robinson, and R. Vidal. Sparse subspace clustering with missing entries. In Proceedings of The 32nd International Conference on Machine Learning, pages 2463-2472, 2015. 